\title{
Agua caliente sanitaria de uso doméstico con Energía Solar, una alter- nativa para la ciudad de Cuenca
}

Ing. John Calle M.Sc; Ing. Jorge Fajardo; Ing. Luis Sánchez jcalle@ups.edu.ec

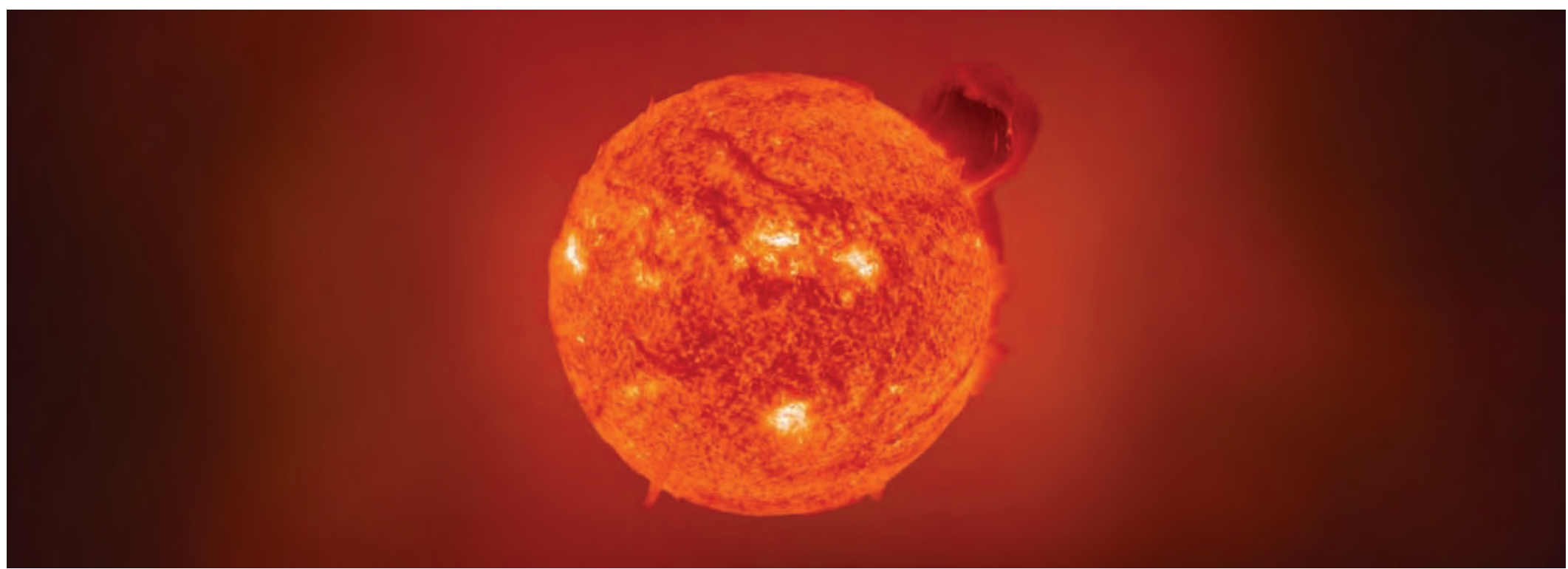

\section{Resumen}

El ACS [Agua Caliente Sanitaria] de uso doméstico es una necesidad imperante debido a las condiciones de temperaturas bajas en la ciudad de Cuenca, para suplir esta necesidad actualmente se utilizan sistemas eléctricos y a base de GLP [Gas licuado de petróleo], los mismos que en su producción y uso emanan dióxido de carbono [CO2] el cual se constituye en el primer factor de contaminación.

La energía solar utilizada a través de colectores solares de tubos de vacío para la obtención de ACS, es una tecnología que aporta significativamente a disminuir la utilización de combustibles fósiles; actualmente en el Ecuador se comercializa colectores de este tipo, que han sido copiados o importados de países europeos y norteamericanos, en donde las condiciones meteorológicas y de localización son diferentes a las que se tiene en Ecuador. En el presente estudio se caracteriza un colector solar de tubos de vacío, a través de la verificación de su eficiencia con los datos ambientales, y de localización medidos en el lugar de emplazamiento y se estructura un diseño con características que favorecen su implementación en la ciudad de Cuenca, es decir considerando su localización, datos meteorológicos y condiciones tecnológicas que posibilitan la fabricación de los mismos en la industria local.

\section{Palabras clave}

Energía solar, colector solar, tubos de vacío, captador solar, agua caliente sanitaria. 


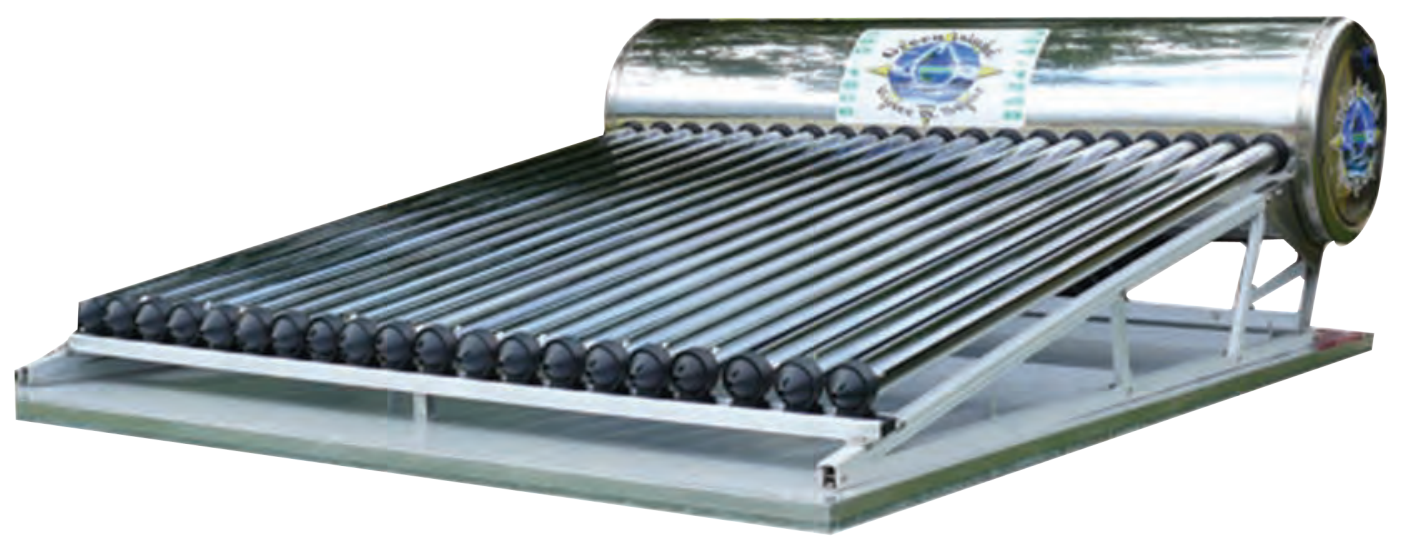

\section{Introducción}

Las tecnologías para el aprovechamiento de la energía solar consisten en la conversión de la radiación solar (fotones) en calor, produciendo un aumento de temperatura conocido como efecto foto-térmico. Este calor suele transferirse y transformarse de muy diversos modos, dando lugar a la serie de tecnologías solares térmicas que conocemos en la actualidad.

Los procesos tecnológicos, desarrollados por el hombre, para el aprovechamiento de la energía solar se basan también en mecanismos fototérmicos y fotoquímicos de conversión y añaden otros mecanismos, como la conversión fotovoltaica.

Las tecnologías basadas en la 'energía solar térmica', forman parte de una de las categorías de energías renovables, la también denominada "uso directo de la energía solar";

\footnotetext{
1
}

Gestión de las energías renovables: Perspectivas de futuro. Quinta Edición, 2007. la misión de la energía solar térmica, junto a las otras energías renovables, es aproximarnos a un desarrollo sostenible, sustituyendo el consumo de recursos fósiles y dar soluciones energéticas en zonas aisladas o no conectadas a las redes de distribución de energías convencionales.

La secretaria de energía renovable y eficiencia energética, de Ecuador, tiene como uno de sus proyectos "El aprovechamiento de energía solar térmica para el calentamiento del agua"; el objetivo principal de este proyecto es la disminución de los egresos que destina el Estado para la comercialización interna de combustibles a partir del aprovechamiento de energía solar térmica para el calentamiento de agua en el sector residencial.

El MEER (Ministerio de Electricidad y Energías Renovables) con este proyecto podría fundamentarse para promover las políticas que permitan desarrollar la oferta y demanda de calentadores solares de agua, la sustitución de 20 mil calefones anuales, establecer la norma- 
tiva que permita asegurar que los nuevos proyectos habitacionales cuenten con las facilidades para instalación de colectores solares que cubra el 100\% de su demanda energética para calentamiento de agua en Industrias, Hoteles y, principalmente, en residencias.

En las ciudades de la sierra, es donde más útil sería un sistema de calentamiento solar para agua, por lo que, al ser el clima frío, es muy difícil que las personas cumplan con las labores de aseo personal con el agua a temperatura ambiente; es por eso que se estima que casi el 100\% de los hogares cuentan con sistemas de calentamiento de agua a partir de electricidad o GLP (calefones).

En el Ecuador se encuentran comercializando algunos tipos de colectores solares, los mismos que no tienen información técnica que identifique sus capacidades y su eficiencia, y que son modelos de países europeos en donde la localización y las condiciones atmosféricas son totalmente diferentes; el proyecto toma como modelo un colector solar de tubos de vacío que se comercializa en el país y lo caracteriza de tal manera que los datos estadísticos obtenidos evalúan su eficiencia y permiten diseñar un sistema que considere las condiciones ambientales y de localización para la ciudad de Cuenca.Además, es importante el análisis técnico financiero que determina parámetros para su desarrollo e implementación.

\section{Materiales y métodos}

El estudio fundamenta su análisis en un colector solar que se comercializa en la ciudad de Quito-Ecuador, el mismo que se emplazó en los predios de la Universidad Politécnica Salesiana de Cuenca, específicamente en la sede del Tecni Club, en la red de abastecimiento de agua para las duchas de los camerinos.(Figura I).

Para la investigación se tomaron datos meteorológicos del sector de emplazamiento y condiciones de temperatura. Al no existir para el Ecuador un mapa solar que establezca con certeza estos parámetros, se utilizarán los datos medidos en el lugar del emplazamiento, los datos considerados son: velocidad de viento, radiación solar, humedad relativa, temperatura ambiente, temperatura del agua al ingreso del colector, temperatura del agua a la salida del colector, temperatura de calentamiento y la temperatura del agua a la salida de las duchas.

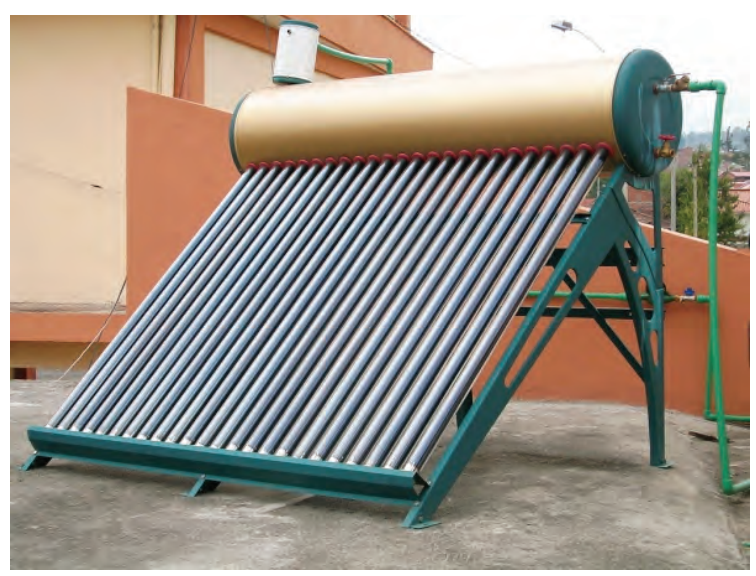

Figura 1. Calentador solar de tubos de vacío.

Para la medición de datos se utilizó un anemómetro portátil Kestrel 4000 Pocket Weather Tracker, un medidor de radiación solar portátil Mac Solar 13236 y sensores de temperatura en los puntos antes indicados, las mediciones se realizaron durante ocho meses, desde junio de 2009 hasta enero de 2010.

Para el análisis estadístico cuando se trata de estudios meteorológicos, se recomienda el uso del método de distribución de frecuencias para el cálculo de la media, por lo que permite procesar la información con mucha facilidad cuando se cuenta con una cantidad de datos muy amplia.

Los resultados obtenidos con las medidas tomadas se muestran en la Tabla I: 
Tabla I. Estadística de datos meteorológicos

\begin{tabular}{|l|c|r|r|r|}
\hline \multicolumn{1}{|c|}{ Variable } & Unidad & Media $(\boldsymbol{\mu})$ & Varianza $\left(\boldsymbol{\sigma}^{2}\right)$ & Desv. Estan. $(\boldsymbol{\sigma})$ \\
\hline Velocidad de viento & $\mathrm{m} / \mathrm{s}$ & 0,66 & 0,05 & 0,22 \\
\hline $\mathrm{T}^{\circ}$ ambiente & ${ }^{\circ} \mathrm{C}$ & 21,85 & 4,28 & 2,07 \\
\hline Radiación & $\mathrm{W} / \mathrm{m} 2$ & 407,95 & 20220,32 & 142,20 \\
\hline Humedad & $\%$ & 47,15 & 64,10 & 8,01 \\
\hline $\mathrm{T}^{\circ}$ entrada colector & ${ }^{\circ} \mathrm{C}$ & 18,75 & 2,62 & 1,62 \\
\hline $\mathrm{T}^{\circ}$ Salida colector & ${ }^{\circ} \mathrm{C}$ & 49,24 & 46,18 & 6,80 \\
\hline $\mathrm{T}^{\circ}$ Duchas & ${ }^{\circ} \mathrm{C}$ & 38,80 & 24,68 & 4,97 \\
\hline
\end{tabular}

Para determinar la densidad de probabilidades de la velocidad de viento, cuando $(v<7[\mathrm{~m} / \mathrm{s}])$ se utiliza la distribución de Weibull (Figura 2) y cuando este valor supera este límite, es decir, $(v \geq 7[\mathrm{~m} / \mathrm{s}])$, entonces se utiliza la distribución de Rayleigh; en nuestro caso, la velocidad media del viento es de 0,79 [m/s], por lo que se utiliza la distribución de Weibull para el cálculo de la probabilidad.
Realizando el análisis correspondiente tenemos:

$$
P(v)=\frac{3.11}{0.79}\left(\frac{v}{0.79}\right)^{3.11-1} e^{-\left(\frac{v}{0.79}\right)^{3.11}}
$$

Esta ecuación permite encontrar la probabilidad de que un valor de velocidad de viento se pueda dar

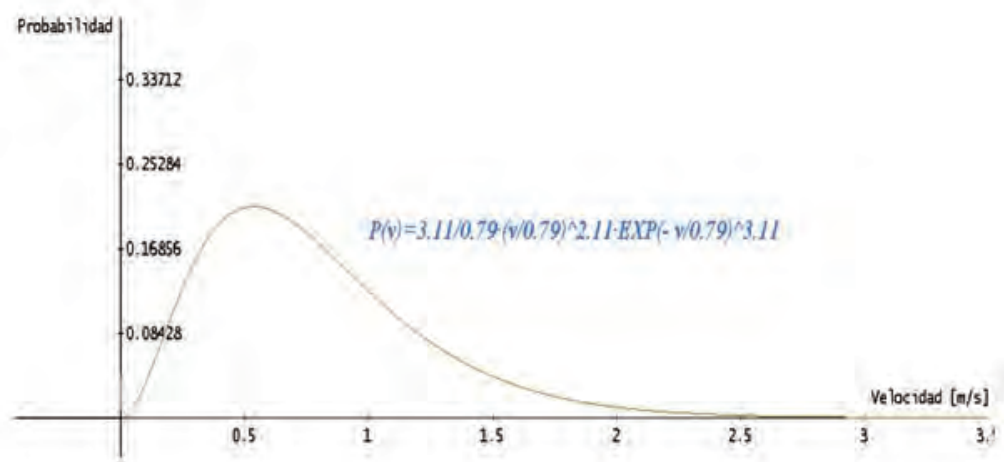

Figura 2. Distribución de Weibull para velocidades de viento

Para calcular la probabilidad de los demás parámetros meteorológicos se va a utilizar el método de la distribución normal y los datos relevantes para el estudio se escriben a continuación:

- La probabilidad de la temperatura ambiente corresponde al 84,I3\% de que la temperatura ambiente sea mayor que $18^{\circ} \mathrm{C}$.

- La probabilidad de la humedad relativa corresponde al $22,36 \%$ de que la humedad relativa sea mayor que $60 \%$, por lo que podemos decir que Cuenca tiene una humedad relativa muy baja.

- La probabilidad de la radiación solar corresponde al 64,06\% de que la radiación solar sea mayor que 300 $\mathrm{W} / \mathrm{m}^{2}$.

Para determinar la eficiencia del colector solar partimos de las especificaciones técnicas del mismo: Figura. 3. 


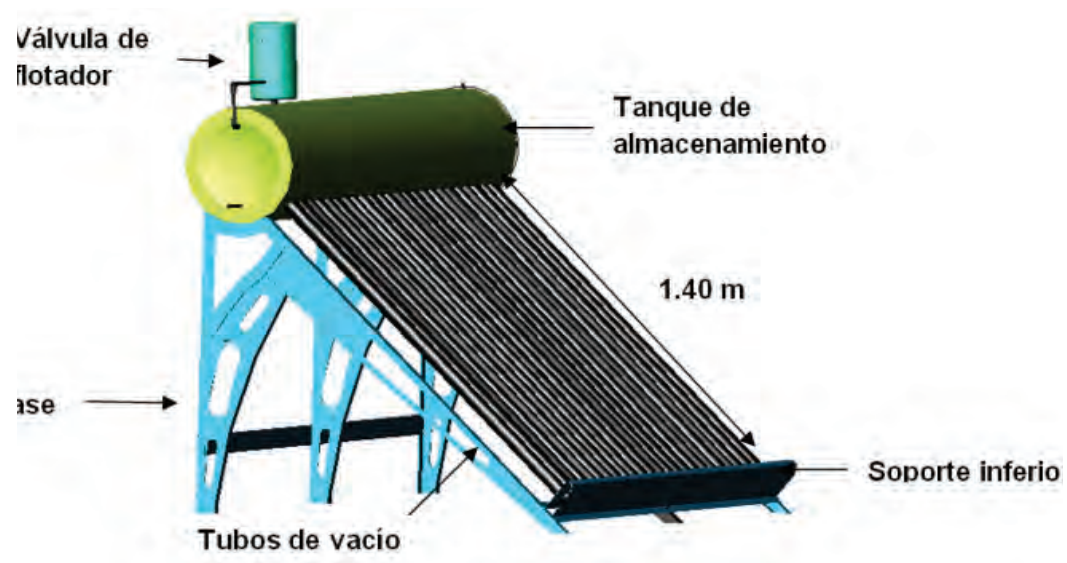

Figura 3. Colector solar

Tanque de almacenamiento: capacidad 130 litros, aislamiento interno: poliuretano de $5,5 \mathrm{~cm}$ de espesor.

Tubos al vacío: cantidad: 24, diámetro exterior del tubo: 0,47 cm., diámetro interior del tubo $0,37 \mathrm{~cm}$, largo: $150 \mathrm{~cm}$.

Interior de Tanque: plancha de acero inoxidable SUS304 / 2B, espesor: 0,4 mm, diámetro: $310 \mathrm{~mm}$

Exterior de Tanque: Plancha de acero pintado, espesor: 0,35 mm, diámetro: $420 \mathrm{~mm}$, longitud: I,7 m.

Para realizar el cálculo se tiene en cuenta las siguientes consideraciones:

- El área de captación en el tubo de vacío es la periferia del tubo interior debido a que es ahí donde se va a calentar el agua, el tubo exterior únicamente mantiene el vacío para que no exista pérdidas por conducción o convección y que las pérdidas por radiación sean mínimas; su superficie siempre está por debajo de la temperatura ambiente.

- Las superficies comunes, como la hierba, los árboles, las rocas y el concreto reflejan alrededor del $20 \%$ de la radiación. Tomando en cuenta que el colector se encuentra sobre una terraza de concreto, el espacio entre tubo y tubo debería considerarse para el cálculo, pero trabajando sólo con el $20 \%$ de la radiación total. Realmente esta consideración debería hacerse sólo para el momento en que el Sol se encuentre totalmente perpendicular al colector, pero, como eso duraría sólo un momento al medio día, entonces se desprecia ese valor.

Para poder conocer la eficiencia del colector solar que es base del estudio, hay que considerar algunos factores:

- El recurso energético o radiación solar con que se cuenta en el sitio donde va a ser emplazado el colector.

- Propiedades físicas de los tubos de vacío: absortividad y emisividad en la placa captadora de nitrato de aluminio que se encuentra adherida a los tubos interiores y transmisividad solar y emisividad de los tubos de vidrio externos de borosilicato.

En estado estable, el funcionamiento de un colector de tubos de vacío se puede describir mediante el siguiente balance de energía:

$$
Q_{\text {incid }}=Q_{u}+Q_{i}+\frac{d u}{d t}
$$

de donde $Q_{\text {incid' }}$ es el calor total incidente absorbido por el colector, $Q_{\text {, }}$, es la energía útil que se transmite al fluido, $Q_{L^{\prime}}$ las pérdidas de calor hacia los alrededores por radiación, convección y conducción, du/dt, es la rapidez del cambio de energía interna almacenada en 
el colector que generalmente se la desprecia por ser un valor muy pequeño.

Para calcular la eficiencia del colector solar de tubos de vacío, se utiliza la siguiente ecuación:

$$
\eta=\frac{Q_{u}}{Q_{\text {incid }}}
$$

Sustituyendo valores calculados con las leyes de la termodinámica y la transferencia de calor, se tiene:

$$
\begin{aligned}
& \eta=\frac{405.37}{549.82} \\
& \eta=0.73 \approx 73 \%
\end{aligned}
$$

Con este resultado se puede establecer que el colector que es objeto de estudio se encuentra trabajando con una eficiencia

\section{promedio de $\mathbf{7 3 \%}$.}

Para un mejor funcionamiento del calentador, es decir, alcanzar la máxima captación solar, sería necesario que los tubos colectores se ubiquen de tal manera que los rayos solares incidan perpendicularmente sobre ellos (Figura 4).

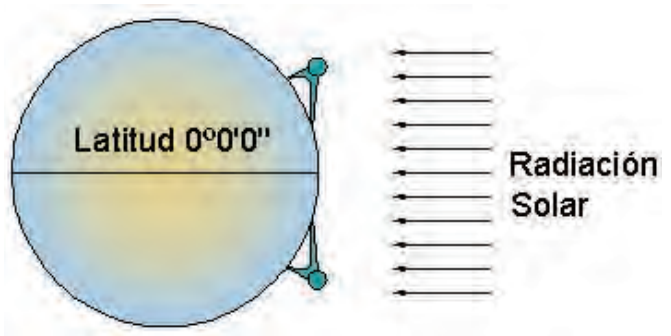

Figura 4. Incidencia de Rayos solares

Sin embargo, como el colector va a estar fijo a una estructura, y la inclinación de los rayos varía en el transcurso del día y con el cambio de las estaciones, entonces es imposible que el colector esté siempre perpendicular a los rayos solares. Para maximizar la radiación se debe conocer la geometría del movimiento solar, y estimar la localización promedio del sol según el tiempo del día y del año en que se utilizará el colector.
Los colectores solares deben estar ubicados según la latitud en donde se quiera emplazar el sistema, si se encuentra en el norte, el colector tiene que estar orientado hacia el sur y si se encuentra en el sur, debe orientarse hacia el norte

Para una captación total de la radiación solar, el colector debería estar en posición horizontal, pero se tendría dificultad con el efecto termosifón, que nos permite la circulación natural dentro del colector. El ángulo correcto para una mejor captación y para poder mejorar el efecto termosifón, determinado mediante pruebas, es de 15 a 20 grados, con respecto a la horizontal, pero tomando en cuenta la corrección por la latitud de ubicación, es decir, se debe sumar el ángulo de latitud².

\section{Resultados y discusión}

Analizando los datos meteorológicos de la zona de emplazamiento se establece que las condiciones para que trabaje el colector son favorables, especialmente en lo que se refiere a radiación solar y temperatura.

La eficiencia del colector en estudio tiene un valor del $73 \%$, si se mejoran algunas situaciones en el diseño la eficiencia mejoraría lo que haría más atractiva la utilización del sistema.

La ciudad de Cuenca se encuentra a una latitud de $2^{\circ} 53^{\prime} 57^{\prime \prime}$ sur, por lo tanto, el colector debe estar orientado hacia el norte y su ángulo de inclinación con respecto a la horizontal debe ser de $\mathbf{2 0}^{\circ}$ (tomando el valor de $17,5^{\circ}$ como referencia en función del efecto termosifón ).

Los tubos de vacío trabajan por el efecto termosifón como se muestra en la Figura 5.

De esta manera, el agua caliente se acumula en el tanque, la cual puede ser utilizada cuando exista necesidad.

Una vez utilizada una cantidad de agua caliente, se llena con agua fría de la red, reiniciándose el movimiento. Para poder contar

Documento del seminario "Las aplicaciones productivas y sustentables de la energía solar" Laboratorio de Energía Solar. Departamento de Física. Universidad Nacional Heredia. Costa Rica. 
con agua caliente para consumo en la noche, es necesario que el tanque de almacenamiento tenga un mínimo de pérdidas y pueda mantener el agua caliente cuando no existe radiación solar.

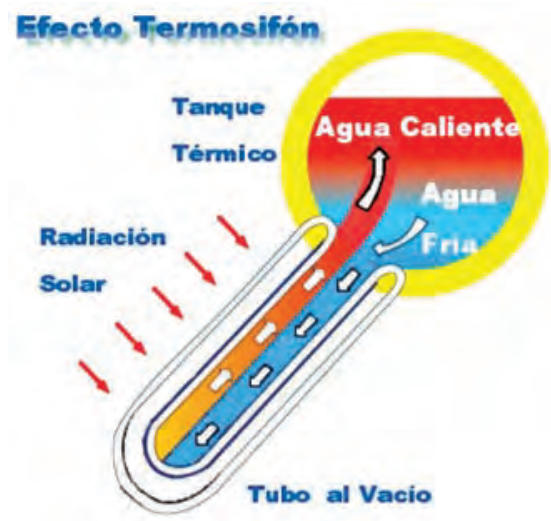

Figura 5. Efecto Termosifón

Otro dato muy importante es el consumo diario que se va a tener, debido que, el colector tiene que ser capaz de abastecer las necesidades de agua caliente en todo el día. Se toma como referencia una familia promedio de 5 personas, en la ciudad de Cuenca el consumo promedio de agua es de 22 a 25 metros cúbicos al mes ${ }^{3}$. El uso de agua caliente en un hogar normalmente está entre el $60 \%$ y $70 \%$ del consumo total ${ }^{4}$.

Para abastecer la demanda de un hogar promedio de 5 personas se diseña el calentador solar con una capacidad de almacenamiento de 300 litros, para lo cual el número de tubos sería 30.

Hay que tomar en cuenta que la radiación en la ciudad es muy variable, es decir, unos días puede presentarse una buena radiación en todo el día, así como también puede ser sólo por unas horas o tener un día totalmente nublado sin presencia de radiación directa. Por ese motivo el tanque de almacenamiento del sistema va a ser de 300 litros, para evitar la carencia de agua caliente por consumo y/o

Ing. Xavier Vidal. Gerente de agua potable. Empresa municipal ETAPA. por falta de radiación solar. Además el tiempo que tarda en llegar a un equilibrio térmico el calentador que se está estudiando es de aproximadamente 2 horas con una radiación solar alta (350 a $\left.600 \mathrm{~W} / \mathrm{m}^{2}\right)$, pudiendo tardar más en ausencia de radiación solar directa.

El objetivo de reducir el ángulo de inclinación lo más posible es para aumentar la capacidad de captación solar.

La representación esquemática de esta situación en un colector solar se representa en la Figura 6.
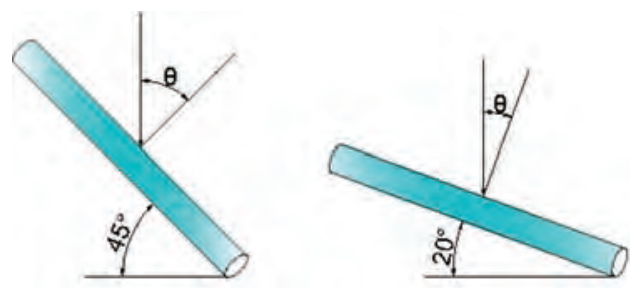

Figura 6. Radiación directa sobre el colector de $45^{\circ}$ y $20^{\circ}$ de inclinación

Analíticamente, al reducir el ángulo de inclinación de los tubos de vacío con respecto a la horizontal de $45^{\circ}$ (ángulo del colector en estudio) a $20^{\circ}$, va a aumentar en un $23 \%$ la capacidad de captación solar, lo que obviamente va a mejorar la eficiencia del colector entre un 15 y un $20 \%$.

En la Figura 7 se presenta el diseño propuesto del colector solar de tubos de vacío en el que las variaciones fundamentales son la capacidad, el número de tubos y el ángulo de captación.

Al realizar un análisis técnico financiero para la implementación de una empresa rentable que fabrique los colectores solares se lograría fabricar 120 colectores al año a un precio de venta al público de $\$ 1,100.00$.

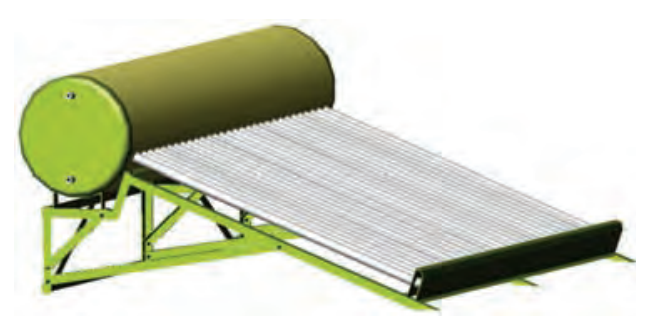

Figura 7. Diseño del colector solar propuesto 


\section{Conclusiones}

Se puede concluir que las condiciones atmosféricas y de temperatura ambiental de la ciudad de Cuenca son favorables para la utilización de colectores solares de tubos de vacío, considerando las diferentes épocas del año.

Los colectores solares de tubos de vacío que se comercializan para el Ecuador no tienen las características necesarias para que trabajen con una eficiencia superior al $80 \%$ ya que fueron diseñados para otra localización y diferentes condiciones ambientales.

El análisis termodinámico y de transferencia de calor en el diseño del nuevo colector abaliza su mejora en eficiencia y el diseño fundamentado en los datos tomados y examinados cubre los requerimientos para la ciudad de Cuenca. Para conseguir aumentos sustanciales en la eficiencia térmica del equipo se lo puede combinar con un sistema auxiliar que provea de la energía adicional en caso de periodos prolongados de nubosidad o de alta demanda de ACS en el hogar.

El costo de estos equipos es bastante elevado en relación a los que utilizamos actualmente a partir de GLP o electricidad, pero este valor se amortiza con el tiempo, debido a que la garantía para un colector solar de tubos de vacío es de 10 años y no representa gastos extras en su utilización.

En la última década se observa un incremento muy grande en el consumo de combustibles fósiles dentro del cantón Cuenca, los cuales constituyen el primer factor de contaminación. Así por ejemplo, el consumo de GLP aumentó un 50\% entre 2002 y 2008 , de seguir la tendencia actual, las emisiones de dióxido de carbono $\left(\mathrm{CO}_{2}\right)$ debidas al consumo de energía pasarán de 190.100 $\mathrm{tCO}_{2}$ anuales en 2006 a $331.250 \mathrm{tCO}_{2}$ en 2017. Un aumento del 74\%. La implementación de fuentes de energía renovables como la presentada en esta investigación aportaría de una manera significativa a reducir estos valores.

El aporte legislativo de la administración nacional y local en el Ecuador es de trascendental importancia para favorecer la implementación de energías alternativas, esto provocaría favorecer la sostenibilidad del ambiente y mejorar las condiciones de vida de las generaciones futuras.

\section{Bibliografía:}

\section{Libros}

I. WALPOLE, Ronald y MYERS, Raymond, Probabilidad y Estadística para Ingeniería y Ciencias, Editorial Pearson Educación, Octava edición, México 2007

2. JANSEN, Ted J, Solar Engineering Technology, Editorial/ production supervision:Albert Boddy and Barbara Palambo

3. CENGEL, Yunus A, "Transferencia de calor", Editorial Mc Graw Hill, Segunda edición, México 2004

4. CENGEL, Yunus A, Termodinámica, Editorial Mc Graw Hill, Cuarta edición, Mexico 2003

5. CUEVAS VILLEGAS, Carlos Fernando, Contabilidad de costos: enfoque general y de gestión, Editorial Pearson Educación, Segunda edición, Colombia 2001 
6. HORNGREN, Charles, DATAR Srikant y FOSTER, George, Contabilidad de costos: un enfoque gerencial, Editorial Pearson Educación, Décima segunda edición, Mexico 2007

7. Fundación Universitaria Iberoamericana, Instituto de estudios Medioambientales, Modulo II de la Maestría en Gestión y Auditorías Ambientales, Aplicación de las Energías Renovables - Energía Solar Térmica , España - 2003

8. FÉLIX TÉLLEZ, Antonio ÁVILA. "Gestión de las Energías renovables, Perspectivas del Futuro - Módulo II Energía Solar térmica", España 2007

9. YÁÑEZ PARAREDA Guillermo, Energía solar, edificación y clima: elementos para una arquitectura solar. Madrid. Ministerio de Obras Públicas y Urbanismo, D.L. 1982

10. Comisión de Gestión Ambiental, I. Municipalidad de Cuenca "Plan Energético de Cuenca 2007-2017", , julio 2007

Il. JOSÉ Ma. DE JUANA, Energías Renovables para el Desarrollo; Madrid, 2003

12. NATHANIEL VANDAL, Wlilliam Herman, Micro-climate Solar Modeling over Complex Terrain, A Validation Study of ESRI Solar Analyst, , Mayo 2007

\section{Documentos electrónicos}

- http://www.martinot.info/reference

- http://www.cordis.lu/eesd/src/ indicators

- http://www.undp.org/seed/eap/ activities/wea/drafts-frame

- http://www.ecologistasenaccion. org/2002/documentos/225_medidas. pdf

- http://editorial.cda.ulpgc.es/ ambiente/2_clima/2_soleamiento/ tesis/soleamie

- http://wrdc-mgo.nrel.gov/html/get_ data-ap

- http://www.eere.energy.gov/

- http://www.solarpaces.org

- http://es.wikipedia.org/wiki/Colector_ solar\#Captadores_de_baja_ temperatura

- http://energiasolar.galeon.com

- http://thales.cica.es/rd/Recursos/rd99/ ed99-0226-0 I

- http://www.natureduca.com/energ_ alternat_eolicaintrod.php

- http://www.greenpeace.org

- http://www.olade.com

- http://www.procobre.org

- http://www.amordad.es

- http://www.textoscientificos. com/energia/calentador-solarl construccion-colectores

- http://www.belenos.es

- http://www.sitiosolar.com 